\title{
Pinning down Notch activation
}

The Notch signalling pathway is deregulated in many cancers; however, the mechanisms that underlie aberrant activation of Notch are not well understood. A recent study shows that the prolyl isomerase PIN1 is an activator of Notch signalling in breast cancer, highlighting a novel molecular circuit that could be targeted for therapeutic benefit.

PIN1 is important in the control of proliferation and PIN1 deregulation has been implicated in oncogenesis. PIN1 interacts with phosphorylated Ser/Thr-Pro motifs leading to conformational changes in its substrates. Because the Notch intracytoplasmic domain (NICD), which when cleaved translocates to the nucleus to regulate transcription, contains many Ser/Thr-Pro motifs, Alessandra Rustighi and colleagues predicted that it might be a PIN1 target. Indeed, PIN1 was found to bind directly to the Ser/Thr-Prorich region of $\mathrm{NOTCH} 1$ in breast cancer cells in a phosphorylationdependent manner. This leads to increased cleavage of NOTCH1 by $\gamma$-secretase, and increased amounts of activated NOTCH1.

The authors observed that deregulated activation of N1ICD caused higher PIN1 protein levels and found that N1ICD binds to the PIN1 promoter, upregulating PIN1 expression. Thus NOTCH1 induction of PIN1 transcription

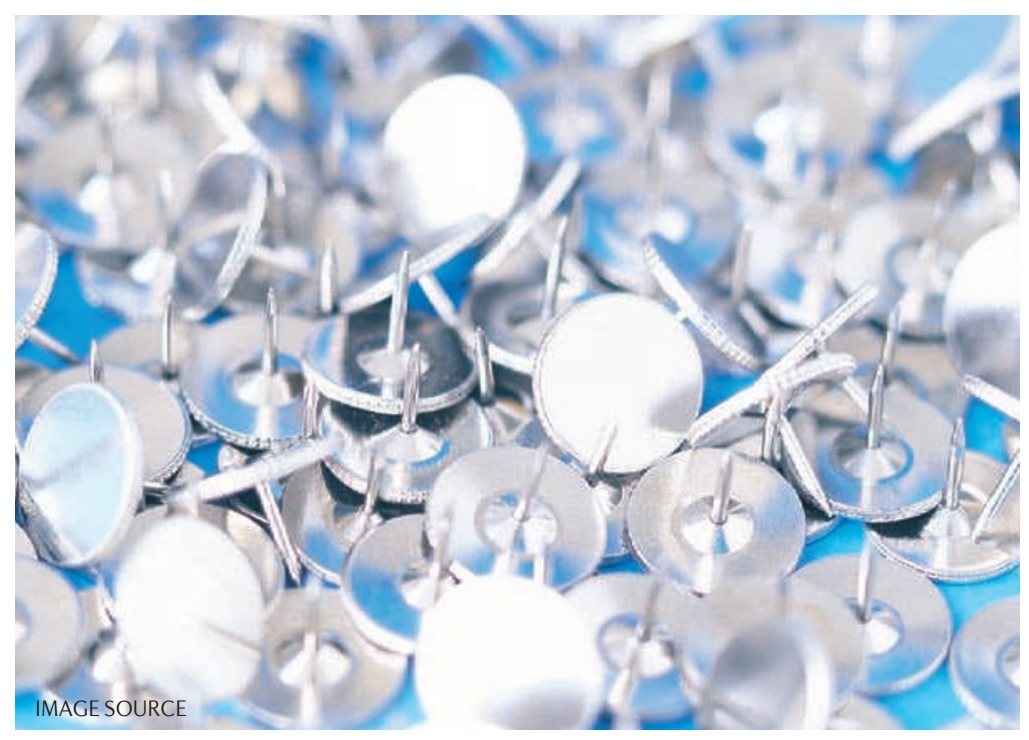

establishes a positive feedback loop. Notably, they observed a strong correlation between high levels of N1ICD and PIN1 in breast cancer cell lines and primary tumours. Furthermore, the NOTCH1-PIN1 molecular circuitry causes transformation of normal breast cells and affects the growth of MDA-MD-231 breast cancer cell xenografts that contain hyperactivated NOTCH1, as PIN1 downregulation by small hairpin RNA impaired tumour growth.

The results of this study could be used to design more effective cancer therapies. Blocking NOTCH1 signalling by targeting $\gamma$-secretase has previously been explored as a treatment. However, although $\gamma$-secretase inhibitors (GSIs) are effective in vitro, they are associated with toxicity in vivo. Rustighi et al. found that inhibiting both NOTCH1 and PIN1 in a breast cancer xenograft mouse model led to synergistic inhibition of tumour growth, suggesting that, in tumours with deregulated NOTCH1 and PIN1, lower doses of GSIs could be combined with PIN1 inhibitors to avoid GSI-associated toxicity.

Meera Swami

ORIGINAL RESEARCH PAPER Rustighi, A. et al The prolyl-isomerase Pin1 is a Notch1 target that enhances Notch1 activation in cancer. Nature Cell Biol. 18 Jan 2009 (doi: 10.1038/ncb1822) FURTHER READING Yeh, E. S. and Means, A. R. PIN1, the cell cycle and cancer. Nature Rev. Cancer 7, 381-388 (2007) 